\title{
Design and Simulation of a Multihoming-based Inter-system Handoff Scheme in NEMO
}

\author{
Shayla Islam ${ }^{1}$, Aisha Hassan Abdalla ${ }^{1}$, Mohamed Hadi Habaebi ${ }^{1}$, Wan Haslina Hassan ${ }^{2}$, \\ Mohammad Kamrul Hasan ${ }^{1}$ \\ ${ }^{I}$ Department of Electrical and Computer Engineering, International Islamic University, \\ Malaysia \\ ${ }^{2}$ Department of Electrical and Computer Engineering, University Technology Malaysia (UTM) \\ iium19612@hotmail.com
}

\begin{abstract}
Network mobility is an innovative concept for conducting a set of mobile nodes within a car-based mobile network. With the expanding wireless technology and increasing demands for ubiquitous Internet connectivity, mobile network is becoming more demanding technology. As a result, network mobility basic support protocol has been proposed by the internet engineering task force for the continual worldwide connectivity of each node within the mobile network. However, applying the multihoming technique in mobile network has become a considerable challenge due to rising traffic load resulting in extra handoff delay during inter system handoff of mobile router in network mobility. Multiple care of address registration mechanism among mobile routers along with its home agent has already been standardized to resolve some of the multihoming issues of network mobility. Yet a flow-based routing mechanism is still needed to support the mobility management in network mobility. Therefore, with the intent of reducing handoff delay, a novel multihoming-based inter-system handoff scheme on proxy mobile IPv6 domain in network mobility is proposed in this paper. Besides, the performance is evaluated through a simulation approach to validate the applicability and efficiency of the proposed scheme.
\end{abstract}

Index Terms-Network mobility, mobile router, multiple care of address, network mobility basic support protocol.

\section{INTRODUCTION}

The idea of using a single address creates the problem of network failure leading to shutdown of Internet connection. This happens only if that specific single interface connection stops and no options remain to keep the connectivity. With the help of multihoming technique, users can switch between more than one interfaces [1]-[4]. To be specific, multihoming is a networking idea which involves simultaneous use of many interfaces or Internet Protocol (IP) addresses on a single Mobile Node (MN) or Mobile Router (MR). This improves the whole Internet connectivity and extends the reliability of network applications. To perform in case of failure at any single attachment point, the functionality of a system unit is taken over by secondary

Manuscript received 25 January, 2015; accepted 9 June, 2015.

A special thanks to the Government of Malaysia, through Ministry of Education (MoE) for the education sponsorship. I would also like to thank Research Management Centre (RMC) at the International Islamic University Malaysia (IIUM) in part for the Endowment Fund grant no. EDW B13-041-0926. system workings if the main unit is not available (e.g. failure). A certain flow can be replicated through various interfaces in few cases. Flow bindings is an extension of Multiple Care of Address (MCoA) in Network Mobility (NEMO) that lets a MR to encompass certain flow to a CoA without affecting other flow through a similar home address [2]. Moreover, it can combine a distinctive flow to a certain $\mathrm{CoA}$ via Correspondent Node (CN) with Home Agent (HA) on a network-based mobility protocol (i.e. Proxy Mobile IPv6) [3], [4]. Hence, it is anticipated to decrease the handoff delay for applications running in real time. Consequently, PMIPv6-based flow mobility solution has already leaded to decrease registration delay during intrasystem handoff [5]. Nevertheless, adding this network-based localized protocol within mobile network for inter-system handoff is still an issue as both MR and its MNs have to be considered. In addition, a dynamic flow redirection mechanism is necessary among the tunnels to solve multihoming problems in NEMO such as prefix ownership and principal CoA registrations of MR with its HA which increases the handoff delay causing higher packet delivery cost and extra signalling overhead [1]-[5]. To avoid this setback, a novel Multihoming-based Inter-system handoff scheme on PMIPv6 domain in NEMO (MI-PNEMO) is suggested in this paper with performance estimation outcomes. It is assured from the outcomes that, MI-PNEMO provides lesser handoff delay at the time of inter-system handoff. The remaining part of the paper is structured as follows: related work on flow mobility scheme in multihomed NEMO is illustrated in Section II. The details of the proposed MI-PNEMO scheme is defined in Section III. Then, the MI-PNEMO scheme is evaluated through simulation approach concerning handoff delay, packet loss and throughput investigation in Section IV and conclusion is given in Section V.

\section{RELATED WORK}

Despite the fact that NEMO-BSP deals with session connectivity and reachability for MNs which are attached to an MR, its handoff performance is often not acceptable. Consequently, the possible cases and issues for NEMO with PMIPv6 have been offered and studied in [6]-[14]. Nevertheless, these techniques integrating PMIPv6 with 
NEMO suffer from extreme packet loss and delay during the operation of inter technology handoff.

Consequently, with the aim of performing mobility as well as traffic management, a router-proxy is adapted as a fundamental gateway inside the NEMO network in [6]. According to this scheme, unidirectional tunnel is used from HA to MR in place of bi-directional tunnel to lessen transmission delay. However, it still need to focus on implementation overhead and security issue.

Multiple MR based scheme in NEMO has considered to improve the band width, network coverage and reliability of a mobile network [7]. Nevertheless, this scheme is not capable of delegating for exclusive downstream communication to MR. So, the authors have proposed a mechanism in addition of the previous scheme that supports both upstream and downstream traffic [8]. The extended strategy permits default router assignment when the MN associates with the moving network. Though, it need to determine the signaling overhead cost of each NEMO entities due to use several MRs.

Multihoming with Intelligent make-before-break in NEMO (MI-NEMO) is a new Infrastructure independent handoff approach that has been developed in [9]. MI-NEMO extends Media Independent Handover (MIH) services to run tunnel establishment and switching before the link break. Thus, the handoff is executed in background with no latency and packet loss while ping-pong scenario is almost avoided. Nevertheless, it is essential to focus on extra signaling cost since the MI-NEMO is a global mobility management based scheme.

Furthermore, a Multihoming-based vertical handoff scheme on PMIPv6 (M-PMIPv6) has been proposed for node mobility in [10]. According to this scheme, access gateway is replaced with a corresponding entity according to access networks. The basic improvement of this scheme is that it supports 'Make-before-break' handoff in network layer with Proxy Binding Update (PBU) messages. However, M-PMIPv6 mechanism must consider in NEMO environment where MR manage the mobility on behalf of all MN.

A Seamless Multi interfaced handoff mechanism in NEMO (SM-NEMO) network is studied in paper [11]. SMNEMO scheme proposed a path selection algorithm to find out the best path among multiple interfaces. It is indicated through analysis that multiple interface-based MR is capable to increase the performance of MR movement during inter technology handoff. Conversely, it is important to focus on the implementation overhead cost for the use multiple interfaces.

Moreover, the authors have proposed some proposals [12]-[14] on flow mobility in PMIPv6 domain to support mobility management. However, the implementation of these proposals with PMIPv6 in NEMO stills an active research area. Moreover, experimental evaluation need to be done to determine the performance of each NEMO entities.

\section{PRoposed MI-PNEMO SCHEME}

Supporting simultaneous Internet connectivity during inter system handoff (i.e., movement of MR from WiFi to $3 \mathrm{G}$ ) in
NEMO can achieve some features such as increased availability and balanced traffic load resulting in lower handoff delay with packet loss [14], [15]. With the intent of getting better throughput for real time applications through utilizing the benefit of all multiple access technologies at any time anywhere in NEMO, MI-PNEMO with predictive mode is proposed in this section (reference scenario is shown in Fig. 1.)

\section{A. Operation of MR, FCMR, FNMR and FLMA}

A Logical Interface (LI) is used by the MR to conceal the current multiple physical interfaces and to support flow based routing in NEMO. The position of LI lies between Layer 3 (L3) and Layer 2 (L2). The LI can simultaneously connect both Flow-based Current MR (FCMR) and Flowbased New MR (FNMR) with the help of multiple interfaces in the proposed MI-PNEMO scheme. The Lower and upper layers forward all the packets through the LI layer. The FNMR and FCMR generally function on an access router and is operated as a substitute of Mobile Access Gateway (MAG) in the proposed MI-PNEMO scheme. FNMR and FCMR is also responsible for detecting the MR's movements among different access technology. Furthermore, the Flow-based Local Mobility Anchor (FLMA) in the proposed scheme functions like a local HA (i.e. Local Mobility Anchor (LMA)) in PMIPv6 [14]. An extension of the FLMA is made to retain several Binding Cache Entries (BCE) for the MR. All entries are assigned for the MR's interface and they also connect with a Primary CoA (PCoA) [14].

\section{B. Handoff Procedure of MI-PNEMO Scheme}

In the proposed MI-PNEMO scheme, it is assumed that all nodes are Local Fixed Node (LFN) underneath the MR and on behalf of LFN, signaling message is fully directed through the MR during inter-system handoff. Otherwise, the MR executes a Network Mobility Basic Support Protocol (NEMO BSP) handoff [14]-[16]. The signaling flow diagram of the proposed algorithm is depicted in Fig. 2.

In accordance with the MI-PNEMO scheme, the Flowbased Proxy Binding Update (FPBU) message is bound up with Handover Initiation (HI) message before the L2 handoff. Hence, it is potential to decrease handoff delay significantly to support mobility management in NEMO The proposed MI-PNEMO handoff scenario is described below in stepwise manner:

Step 1: At first, the MR is linked to the FCMR using the Physical Interface1 (PI 1) and delegates Home Network Prefix 1(HNP1). When MR is nearby the access link of FNMR, then it will make a decision to bind with FNMR using the Physical Interface 2 (PI 2), though it is still connected with FCMR by PI 1.

Step 2: With taking the help of L2 triggering mechanism, two messages (i.e. DeRegistration Proxy Binding Update (DeRegPBU) and DeRegistration Proxy Binding Acknowledgement (DeRegPBA)) are exchanged in between FCMR and FLMA to inform FLMA by FCMR about the handoff detachment of MR from PI 1 to PI 2.

Step 3: FLMA has to wait to execute handoff registration of MR with FNMR after passing DeReg.PBA message to 


\section{FCMR.}

Step 4: After receiving the L2 link up from the FNMR, the authentication procedure is executed by the MR to access the new network. FNMR then waits for Router Solicitation (RS) message from the newly connected MR on its access link while passing the packets from the FNMR to the MR.

Step 5: After receiving the RS message, FNMR sends HI message with the coupled FPBU message (should set $F=1$ ) to FLMA for reporting the presence of MR movement to support flow based routing on PMIPv6 domain as shown in Fig. 3.

Step 6: As the MR supports flow based routing, FLMA transmits the Flow-based Proxy Binding Acknowledgement (FPBA) message (contains ID of MR, HNP-1, and HNP-2 with the MNP) to the FNMR that supports flow bindings as well. As the bidirectional tunnel among the FLMA and FNMR is established, data packets for the MR travels through this tunnel.

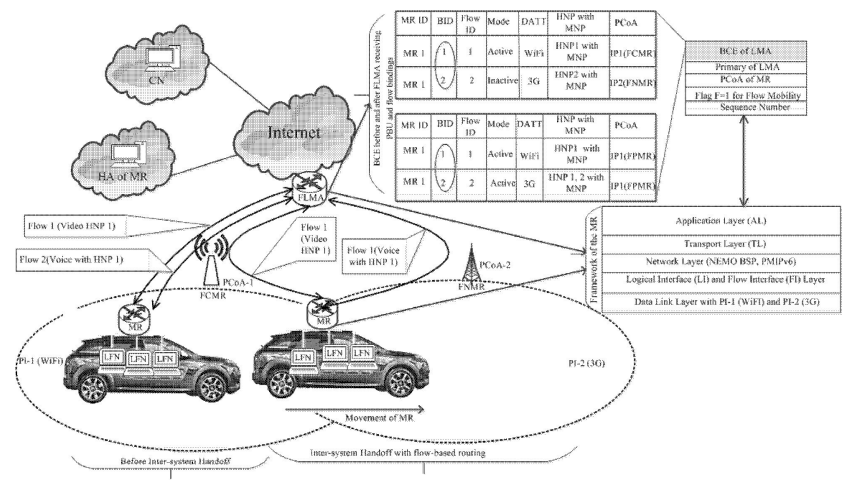

Fig. 1. Reference Scenario for the proposed MI-PNEMO scheme.

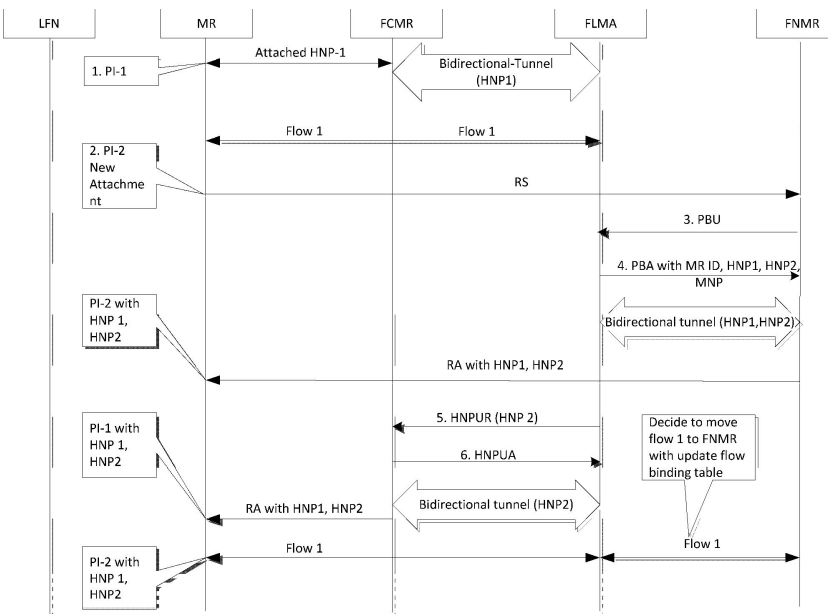

Fig. 2. Signaling Flow Diagram for MI-PNEMO scheme.

Step 7: As soon as the FLMA decides to transmit a flow via HNP, FLMA at first confirms whether HNP can be used on the FNMR or not by scanning the current BCE of the MR. If it can be used, the FLMA should then update the BCE list. If unusable, an HNP Update Request (HNPUR) and HNP Update Acknowledgement (HNPUA) message is interchanged between FCMR and FLMA to notify the HNP used through that flow by forming a bidirectional tunnel. Simultaneously, the corresponding BCE is revised by the FLMA. Consequently, the FLMA is able to move packet flows among multiple interfaces freely with no excess signalling overhead. Hence, it is possible for the FLMA to transmit the flow-1 that uses HNP1, from FCMR to FNMR without deviation.

\section{Extended FPBU Message}

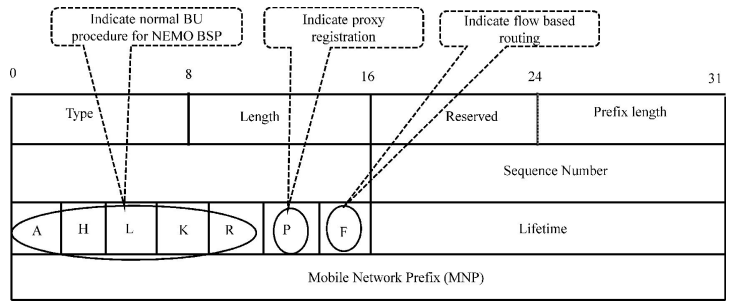

Fig. 3. Extended FPBU Message.

In accordance with the MI-PNEMO scheme, some flags need to be included in FPBU message as shown in Fig. 3. To support the MI-PNEMO scheme, $R$ bit is specified as a flag for defining MN or MR movement [14]-[16]. Flag $F$ is used in the FPBU massage to represent the FLMA that the MR can retain flow based routing. When $F$ flag is set to 1 , the MR will execute flow mobility. Whereas, when it is set to 0 , the MR cannot support flow mobility.

\section{PERFORMANCE ANALYSIS}

The performance of the MI-PNEMO scheme is evaluated by Network Simulator version 3.12.1 (NS 3.12.1). The basis of this simulation is to verify the practicability and efficiency of the proposed MI-PNEMO scheme. In the proposed MIPNEMO scheme, MR and FLMA are extended with PMIPv6 in NS 3.12.1. With lower handoff delay and packet loss can achieve maximum throughput to provide uninterrupted Internet connectivity for real time application scenarios [14]-[17]. Therefore, the performance evaluation is carried out mainly based on significant metrics such as throughput, handoff delay and packet loss respectively.

\section{A. Simulation Framework}

The simulation scenario of the proposed scheme is shown in Fig. 4 in which PMIPv6 is used with WiFi and 3G interfaces network environment. Initially, the $\mathrm{MR}$ is connected to the WiFi network while the $3 \mathrm{G}$ is idled. Three non-identical scenarios are defined to evaluate the proposed MI-PNEMO scheme which are given in Table I.

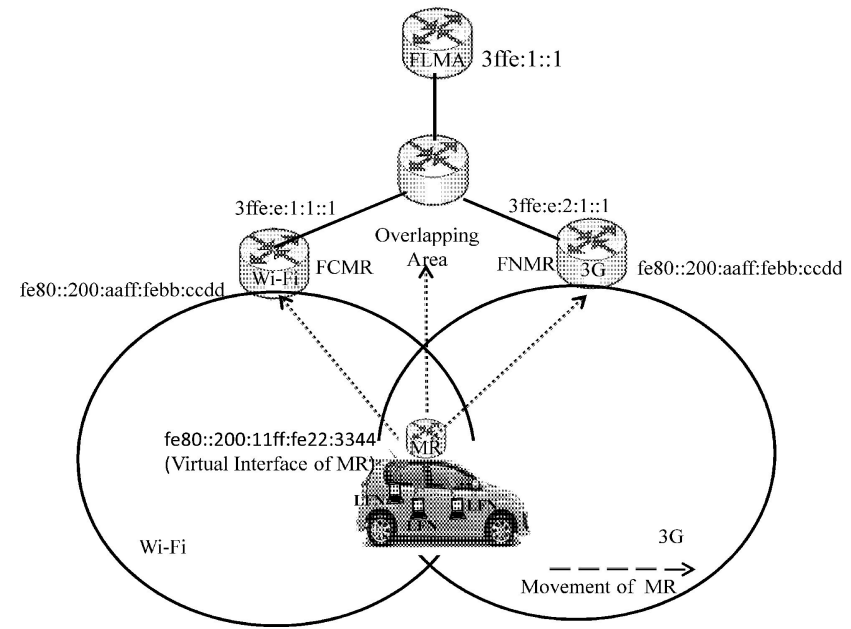

Fig. 4. Simulation Framework for MI-PNEMO scheme. 


\section{B. Result Analysis}

The simulation outcomes are illustrated in Fig. 5-Fig. 7 respectively. The variation of average handoff delay for three scenarios are illustrated in Fig. 5 as a function of the different number of MR. It is observed from Fig. 5, that the MI-PNEMO scheme (i.e. scenario 2) has a lesser handoff delay for the real time scenario.

TABLE I. PARAMETERS FOR HANDOFF DELAY ANALYSIS.

\begin{tabular}{|c|c|}
\hline Step & Phenomenon \\
\hline Scenario 1 & $\begin{array}{l}\text { Activate Wi-Fi Interface while deactivate WiMAX } \\
\text { interface }\end{array}$ \\
\hline $\begin{array}{c}\text { Scenario } 2 \\
\text { (Proposed MI- } \\
\text { PNEMO scheme) }\end{array}$ & $\begin{array}{c}\text { Both interfaces (i.e. WiMAX and Wi-Fi) are } \\
\text { activated. } \\
\text { Flow handoff is initiated by FLMA }\end{array}$ \\
\hline Scenario 3 & $\begin{array}{c}\text { Both Interfaces (i.e. WiMAX and Wi-Fi) are } \\
\text { activated. } \\
\text { Flow handoff is initiated by MR }\end{array}$ \\
\hline
\end{tabular}

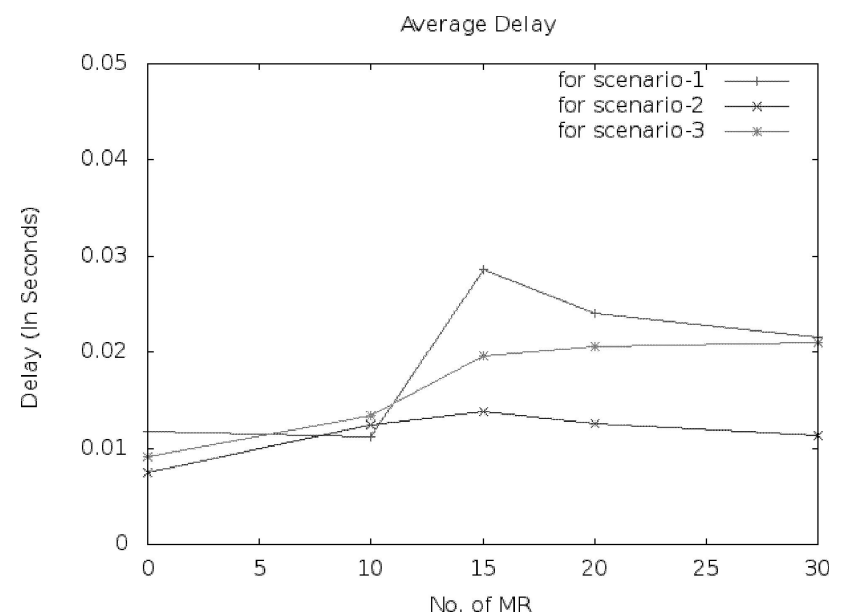

Fig. 5. Handoff Delay of the proposed MI-PNEMO scheme.

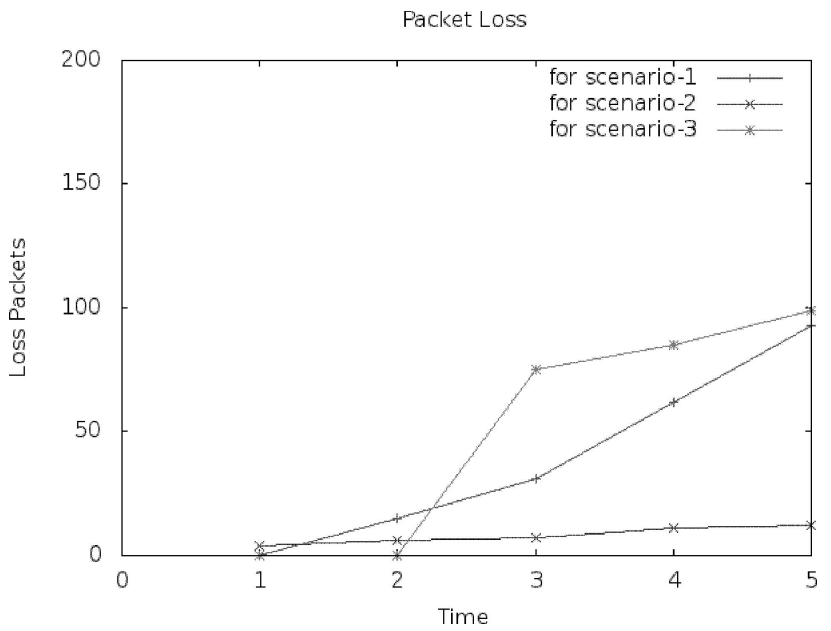

Fig. 6. Packet loss for the proposed MI-PNEMO scheme.

It is indicated from Fig. 5 that, handoff delays of all schemes escalates linearly with raising the number of MR from 10 to 30. Yet, the proposed MI-PNEMO scheme shows improved performance as the number of handoff is reduced through applying early registration mechanism on networkbased mobility protocol. In addition to that, the handoff delay of the proposed MI-PNEMO scheme has become smaller than scenario 1 and scenario 3 due to use multiple interfaces simultaneously during inter-system handoff with initiation of flow handoff by the network side (i.e. FLMA).
As there is a relation among handoff delay and its impact on packet loss, hence the variation of packet loss is shown in Fig. 6 for the proposed MI-PNEMO, scenario 1 and scenario 3 respectively. Eventually, it can be concluded from Fig. 6 that splitting data flow among multiple interfaces simultaneously at the overlapping region during handoff has positive impact on the packet loss for the proposed MIPNEMO scheme. Moreover, it is noticeable from Fig. 6 that, the frequent handoff of the MR in proposed MI-PNEMO scheme is not prerequisite as the local mobility is handled by the local home agent (i.e. FLMA).

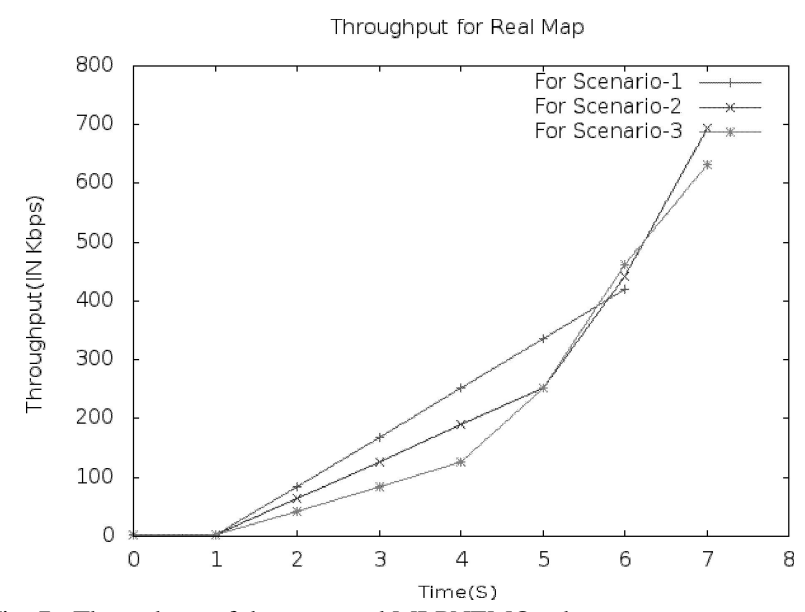

Fig. 7. Throughput of the proposed MI-PNEMO scheme.

Throughput is defined as the complete number of data flows transmitted over the entire period of simulation. As higher throughput indicates better connectivity, hence, it is clearly observed from Fig. 7 that, the proposed MI-PNEMO scheme takes out superior performance than scenario 1 and scenario 3. Therefore, it is summed up from Fig. 5-Fig. 7 that, using multihoming technique on PMIPv6 domain in NEMO can consume network resources efficiently.

\section{CONCLUSION}

This paper proposes a novel multihoming-based interhandoff scheme on PMIPv6 domain in NEMO to support mobility management. The major contribution of this paper is to reduce handoff delay by including PMIPv6 in NEMO. In addition to that, through applying a dynamic flow redirection mechanism, it is feasible to reduce handoff delay which leads to provide a better Quality of Service (QoS). The performance of the proposed MI-PNEMO scheme is evaluated via NS 3.12.1 simulator. Hence, it can be summed up from simulation results analysis that, the throughput of MI-PNEMO shows better improvements than single interface-based scheme. Experimental test bed is recommended as future work for more precise evaluation on end-to-end delay of the proposed MI-PNEMO scheme.

\section{REFERENCES}

[1] B. Sarikaya, "PMIPv6 multihoming support extensions for flow mobility", Internet Draft, 2012.

[2] G. Tsirtsis, H. Soliman, N. Montavont, G. Giaretta, K. Kuladinithi, "Flow bindings in mobile IPv6 and network mobility (NEMO) basic support", RFC 6089, 2011

[3] M. Calderon, C. Bernardos, I. Soto, "PMIPv6 and network mobility problem statement", Internet Draft, 2012. 
[4] V. Devarapalli, R. Wakikawa, A. Petrescu, P. Thubert, "Network mobility (NEMO) basic support protocol", RFC 3963, 2005.

[5] J. H. Lee, T. Ernst, N. Chilamkurti, "Performance analysis of PMIPv6-based network mobility for intelligent transportation systems", IEEE Trans. Vehicular Technology, vol. 61, no. 1, pp. 7485, 2012. [Online]. Available: 10.1109/TVT.2011.2157949

[6] Z. Slimane, M. Feham, A. Abdelmalek, "A seamless and transparent MN-Proxy based mobility support for (n, n, 1) multihomed NEMO model", Int. Journal of Computer Science and Network Security, vol. 10, no. 4, pp. 306-313, 2010.

[7] R. Kuntz, J. Montavont, T. Noel, "Multiple mobile routers in nemo: How neighbor discovery can assist default router selection", in Proc. IEEE 19th Int. Symposium on Personal, Indoor and Mobile Radio Communications, Cannes, 2008, pp. 1-6. [Online]. Available: http://dx.doi.org/10.1109/pimrc.2008.4699613

[8] R. Kuntz, J. Montavont, T. Noel, "Multihoming in IPv6 mobile networks: progress, challenges, and solutions", IEEE Communications Magazine, vol. 51, no. 1, pp. 128-135, 2013. [Online]. Available: 10.1109/MCOM.2013.6400449

[9] Z. Slimane, M. Feham, A. Abdelmalek, "Seamless infrastructure independent multi homed NEMO handoff using effective and timely IEEE 802.21 MIH triggers", Int. Journal of Wireless \& Mobile Networks (IJWMN), vol. 4, no. 3, pp. 119-139, 2012. [Online]. Available: $\quad 10.5121 /$ ijwmn.2012.4308

[10] H. D. Park, K. N. Park, "A multihoming-based vertical handover scheme", Lecture Notes in Electrical Engineering, vol. 215, pp. 749754, 2013. [Online]. Available: 10.1007/978-94-007-5860-5_89

[11] X. Chen, H. Zhang, Y. C. Chang, H. C. Chao, "Experimentation and performance analysis of multi-interfaced mobile router scheme", Simulation Modelling Practice and Theory, vol. 18, no. 4, pp. 407415, 2010. [Online]. Available: doi:10.1016/j.simpat.2009.09.005

[12] T. M. Trung, Han, Y. H. Choi, H. Y. Geun, "A design of networkbased flow mobility based on proxy mobile IPv6", in Proc. IEEE Conf. computer communications workshops (INFOCOM WKSHPS), 2011, pp. 373-378.

[13] T. Melia, C. J. Bernardos, A. De la Oliva, A. F. Giust, M. Calderon, "IP flow mobility in PMIPv6 based networks: solution design and experimental evaluation", Wireless Personal Communications, vol. 61, no. 4, pp. 603-627, 2011. [Online]. Available: 10.1007/s11277-011-0423-3

[14] H. Y. Choi, S. G. Min, Y. H. Han, R. Koodli, "Design and simulation of a flow mobility scheme based on proxy mobile IPv6", Journal of Information Processing Systems (JIPS), vol. 8, no. 4, pp. 603-620, 2012. [Online]. Available: 10.3745/JIPS.2012.8.4.603

[15] C. W. Lee, M. C. Chen, Y. S. Sun, "A novel network mobility management scheme supporting seamless handover for high-speed trains", Computer Communications, vol. 37, pp. 53-63, 2014. [Online]. Available: 10.1016/j.comcom.2013.09.009

[16] Z. Huang, Y. Yang, H. Hu, K. C. Lin, "A fast handover scheme based on multiple mobile router cooperation for a train-based mobile network", Int. Journal of Modelling, Identification and Control, vol. 10, no. 3, pp. 202-212, 2010. [Online]. Available: http://dx.doi.org/10.1504/IJMIC.2010.034572

[17] Y. Chen, T. Farley, N. Ye, "QoS requirements of network applications on the Internet", Information, Knowledge, Systems Management, vol. 4, no. 1, pp. 55-76, 2004. 